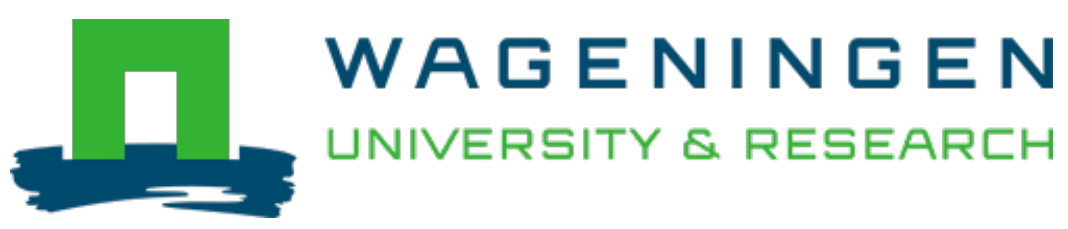

\title{
Meiotic recombination profiling of interspecific hybrid F1 tomato pollen by linked read sequencing
}

\author{
The Plant Journal \\ Rommel Fuentes, Roven; Hesselink, T.; Nieuwenhuis, Ronald; Bakker, L.V.; Schijlen, E.G.W.M. et al \\ https://doi.org/10.1111/tpj.14640
}

This article is made publicly available in the institutional repository of Wageningen University and Research, under the terms of article $25 \mathrm{fa}$ of the Dutch Copyright Act, also known as the Amendment Taverne. This has been done with explicit consent by the author.

Article 25 fa states that the author of a short scientific work funded either wholly or partially by Dutch public funds is entitled to make that work publicly available for no consideration following a reasonable period of time after the work was first published, provided that clear reference is made to the source of the first publication of the work.

This publication is distributed under The Association of Universities in the Netherlands (VSNU) 'Article $25 \mathrm{fa}$ implementation' project. In this project research outputs of researchers employed by Dutch Universities that comply with the legal requirements of Article $25 \mathrm{fa}$ of the Dutch Copyright Act are distributed online and free of cost or other barriers in institutional repositories. Research outputs are distributed six months after their first online publication in the original published version and with proper attribution to the source of the original publication.

You are permitted to download and use the publication for personal purposes. All rights remain with the author(s) and / or copyright owner(s) of this work. Any use of the publication or parts of it other than authorised under article $25 \mathrm{fa}$ of the Dutch Copyright act is prohibited. Wageningen University \& Research and the author(s) of this publication shall not be held responsible or liable for any damages resulting from your (re)use of this publication.

For questions regarding the public availability of this article please contact openscience.library@,wur.nl 


\section{Meiotic recombination profiling of interspecific hybrid F1 tomato pollen by linked read sequencing}

Roven Rommel Fuentes' ${ }^{1}$, Thamara Hesselink ${ }^{2}$, Ronald Nieuwenhuis ${ }^{2}$, Linda Bakker ${ }^{2}$, Elio Schijlen ${ }^{2}$, Willem van Dooijeweert ${ }^{3}$, Sara Diaz Trivino ${ }^{2}$, Jorn R. de Haan ${ }^{4}$, Gabino Sanchez Perez ${ }^{4}$, Xinyue Zhang ${ }^{5}$, Paul Fransz ${ }^{5}$, Hans de Jong ${ }^{6}$, Aalt D. J. van Dijk ${ }^{1,7}$ D, Dick de Ridder ${ }^{1}$ and Sander A. Peters ${ }^{2, *}$

${ }^{1}$ Bioinformatics Group, Wageningen University and Research, Droevendaalsesteeg 1, 6708 PB Wageningen,

The Netherlands,

${ }^{2}$ Business Unit of Bioscience, Cluster Applied Bioinformatics, Wageningen University and Research, Droevendaalsesteeg

1, 6708 PB Wageningen, The Netherlands,

${ }^{3}$ Centre for Genetic Resources, Wageningen University and Research, Wageningen, Droevendaalsesteeg 1, 6708 PB

Wageningen, The Netherlands,

${ }^{4}$ Genetwister Technologies B.V., Nieuwe Kanaal 7b, 6709 PA Wageningen, The Netherlands,

${ }^{5}$ Swammerdam Institute for Life Sciences, University of Amsterdam, Science Park 904, 1098 XH Amsterdam, The Netherlands,

${ }^{6}$ Laboratory of Genetics, Wageningen University and Research, Droevendaalsesteeg 1, 6708 PB Wageningen, The Netherlands, and

${ }^{7}$ Biometris, Wageningen University and Research, Droevendaalsesteeg 1, 6708 PB Wageningen, The Netherlands

Received 24 September 2019; revised 25 November 2019; accepted 4 December 2019.

*For correspondence (e-mail sander.peters@wur.nl).

\section{SUMMARY}

Genome wide screening of pooled pollen samples from a single interspecific F1 hybrid obtained from a cross between tomato, Solanum lycopersicum and its wild relative, Solanum pimpinellifolium using linked read sequencing of the haploid nuclei, allowed profiling of the crossover (CO) and gene conversion (GC) landscape. We observed a striking overlap between cold regions of $\mathrm{CO}$ in the male gametes and our previously established F6 recombinant inbred lines (RILs) population. COs were overrepresented in non-coding regions in the gene promoter and 5'UTR regions of genes. Poly-A/T and AT rich motifs were found enriched in $1 \mathrm{~kb}$ promoter regions flanking the CO sites. Non-crossover associated allelic and ectopic GCs were detected in most chromosomes, confirming that besides CO, GC represents also a source for genetic diversity and genome plasticity in tomato. Furthermore, we identified processed break junctions pointing at the involvement of both homology directed and non-homology directed repair pathways, suggesting a recombination machinery in tomato that is more complex than currently anticipated.

Keywords: recombination bin mapping, tomato, crossover recombination, gene conversion, S. pimpinellifolium, recombination hot spots, recombination cold spots.

\section{INTRODUCTION}

Meiotic recombination is a key fundamental biological process that ensures balanced chromosome distribution and the reshuffling of parental alleles into new combinations. In the current model, meiotic recombination is initiated by the formation of double-stranded DNA breaks (DSBs) that are processed to single stranded DNA, followed by single strand invasion into the intact sister chromatid or one of its non-sister chromatids. Upon break repair on the nonsister chromatids, DSBs are resolved either into crossovers (COs) or non-crossovers (NCOs). The resolution involves homology directed repair (HDR) by the ZMM and non-
ZMM pathways leading to class I and class II COs respectively, and alternative pathways such as synthesisdependent strand annealing (SDSA), dissolution of double Holiday junction $(\mathrm{dHJ})$ and other pathways leading to NCOs (Mercier et al., 2015). In general, CO involves reciprocal exchange of large DNA fragments, which can size up to whole chromosome arms. In contrast, a NCO usually comprises a small patch that is copied from the intact homologous chromosome (Mercier et al., 2015). When the sister chromatid template is used as donor, the repair machinery restores the duplex. When the non-sister chromatid 
template is used as a donor, sequence divergence at the site of strand invasion may give rise to heteroduplex formation. The mismatch repair machinery recognizes and corrects meiotic heteroduplex DNA (Borts et al., 2000), leading to a non-reciprocal exchange of genetic information or gene conversion (GC). Gene conversion can occur between donor and acceptor alleles of the same gene (allelic GC) or between different repetitive loci, the latter known as ectopic gene conversion (non-allelic GC). (Puchta, 2005; Duret and Galtier, 2009; Wijnker et al., 2013; Trombetta et al., 2016).

In plant breeding, the allelic reshuffling and gene conversion via meiotic recombination is a source of genetic diversity exploited by breeders that seek out new allele combinations for trait selection and crop improvement. As assorted alleles determine the inherent properties in progeny, it is important to precisely introgress chromosomal regions of interest, maintaining favorable genes yet preventing other regions harboring undesirable alleles to be recombined. Detection of genetic differences and monitoring of specific characters in segregating populations to assist trait selection is therefore crucial to successful breeding. Efficient detection and precise delineation of recombined alleles has seen many strategies. Microscopic analysis of meiotic events in tomato has provided valuable information on chromosome configurations (Moens, 1964; Havekes et al., 1994; Anderson et al., 2010a,b), but the reduced throughput and low resolution limits genome wide and accurate measurements of recombined alleles. Currently, prevailing technologies to profile segregating alleles in populations include molecular marker-based screenings. In combination with high-throughput genome sequencing, such technologies are extremely powerful to delineate recombined alleles. Furthermore, such screenings provide valuable information on recombination frequency and genetic distances, which is used for genetic linkage map construction and marker assisted breeding. Previously, we profiled recombination events in a RIL population from a cross between tomato and its close relative S. pimpinellifolium, using genome wide SNP analysis. The analysis provided insight into 'hot' and 'cold' regions of recombination, and genomic features involved in crossover recombination (Aflitos et al., 2015; Demirci et al., 2017, 2018). However, these methods require the production of offspring populations involving several generations and subsequent genetic marker screening for a large number of progeny plants (Paran et al., 2004; Van Os et al., 2006; Ganal et al., 2011; Baurens et al., 2019). Often marker coverage is limited, causing low genetic resolution, and when integrated with physical maps, allows detection of crossovers in the kilo base range at best. Markers may even be absent leaving substantial genome portions uncovered and thus opaque to further genetic profiling. Furthermore, the accurate detection of $\mathrm{COs}$ and GCs by
NGS technologies has long been hampered by short read length limitations, a relatively high base call error rate for long reads, and/or inadequate bioinformatics solutions. Therefore, more affordable quantitative methods to study $\mathrm{CO}$ and GC distributions are desired.

Here we present a method for profiling meiotic recombination in pollen nuclei from a single F1 interspecific hybrid between tomato and the closely related $S$. pimpinellifolium. The identification of polymorphisms marking COs and GCs is greatly improved by high-throughput long read sequencing with high base call accuracy, facilitating unambiguous read mapping and accurate identification of haplotype phases. We pinpoint COs and GCs at the SNP resolution level, using 10X Genomics linked read sequencing of gDNA from F1 pollen nuclei and subsequent detection of phase shifts in large gDNA molecules, providing further insight into gross similarities of recombination. Our sequence based approach can be applied to reliably profile meiotic recombination in a wide variety of crop species without the laborious and time-consuming production and screening of offspring populations, greatly benefitting introgression hybridization and precision breeding.

\section{RESULTS}

\section{Detection of haplotype shifts and CO designation}

Our aim was to localize recombination events at SNP resolution, taking advantage of linked-read sequencing of an F1 hybrid pollen grain population and the high density of informative polymorphisms between $S$. Iycopersicum Heinz 1707 and S. pimpinellifolium CGN14498 parental genomes, allowing a detailed analysis of genomic sequences involved in $\mathrm{CO}$ recombination. To this end we constructed a linked read sequencing library from $\sim 0.70 \mathrm{ng}$ input gDNA and produced $781.3 \times 10^{6}$ paired end (PE) linked reads. With a median insert size of $391 \mathrm{bp}$, the data represented a mapped genome coverage of about $68 \times$. Approximately $60 \%$ and $18 \%$ of the input gDNA molecules was longer than 20 and $100 \mathrm{~kb}$, respectively, with a mean molecule length of $25.5 \mathrm{~kb}$. We obtained $1.61 \times 10^{6} \mathrm{gel}$ beads in emulsion (GEMS) with a mean DNA molecule length per GEM of $285 \mathrm{kbp}$, and an N50 linked reads per molecule (LPM) of 12. Given these GEM performance statistics, the molecule coverage for each genome copy was approximately $86 \%$. Using the LONG RANGER pipeline from 10X Genomics, approximately $95.7 \%$ of the reads was successfully mapped to the tomato reference genome, leaving less than $0.14 \%$ of the reference bases uncovered. We detected polymorphisms in S. pimpinellifolium and $S$. lycopersicum PE reads that were aligned to the tomato SL3.0 reference genome, using SNP callers from CLC and GATK (McKenna et al., 2010). Approximately $4.65 \times 10^{6}$ CLC and $4.90 \times 10^{6}$ GATK $S$. pimpinellifolium homozygous SNP calls were intersected, based on their corresponding 
genomic position, rendering $4.05 \times 10^{6}$ high quality homozygous SNPs (Figure S3). To remove possible base call errors and individual-specific sequence differences, approximately 210000 SNPs between the tomato parent and the reference genome were excluded, leaving $3.84 \times 10^{6}$ homozygous SNPs at a SNP density level of 1 per $210 \mathrm{bp}$. These SNP levels are in agreement with the SNP density level of $0.58 \%\left(\sim 3.89 \times 10^{6} \mathrm{SNPs}\right)$ as previously determined in genetic diversity study for the same parental lines (Aflitos et al., 2014).

The SNP profiles in the genomic DNA molecules enabled us to assign COs according to the observed haplotype phase shifts. We first filtered ambiguous mappings, discarding discordant reads and molecules significantly overlapping with tomato genome repeats. Additionally, we removed recombinant molecules containing phase shifts with a skewed read coverage, that may have arisen from unknown repeat elements or copy number variation. Furthermore, we rejected multiple haplotype shifts, thereby avoiding cases where multiple putative $\mathrm{COs}$ or GCs occurred in the same molecule. These filtering constraints rendered a total of 5126 recombinant molecules on 12 chromosomes. We also anticipated on the occurrence of possible non-crossover associated (NCO-GC) and CO-associated gene conversions (CO-GCs) as possible products of recombination events. Indeed, a previous study on targeted recombination analysis in tomato reported on a single discontinuous GC of 5-6 kb. This GC however, was observed in somatic tissue with artificially induced double stranded breaks (DSBs) by Cas9 (Filler Hayut et al., 2017). In rice the average length of a gene conversion was reported to be $130 \mathrm{bp}$ (Xu et al., 2008). Considering that the observed average length of NCO-GCs and CO-GCs in A. thaliana ranges between $25-400 \mathrm{bp}$ (Wijnker et al., 2013), the additional filtering for tomato GCs left 3819 recombinant molecules representing candidate $\mathrm{CO}$ regions with haploblock sizes larger than 400 bp, a resolution (i.e. distance between markers flanking a $\mathrm{CO}$ ) ranging from $2 \mathrm{bp}$ to $213 \mathrm{~kb}$, and spanning distance (i.e. between the first and last markers within a recombinant molecule)

(a)

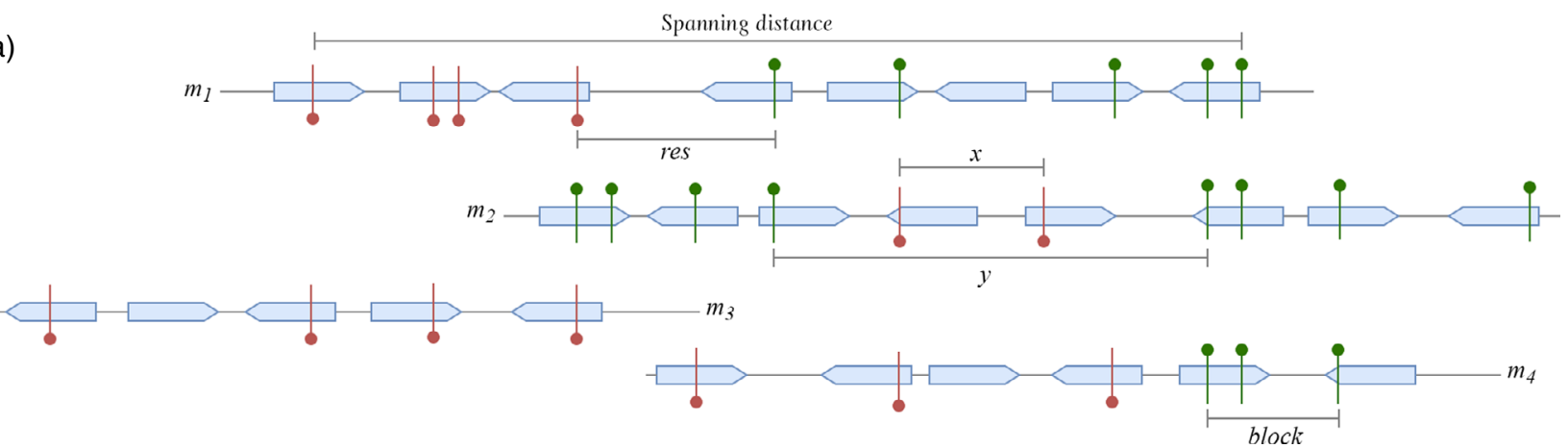

(b)

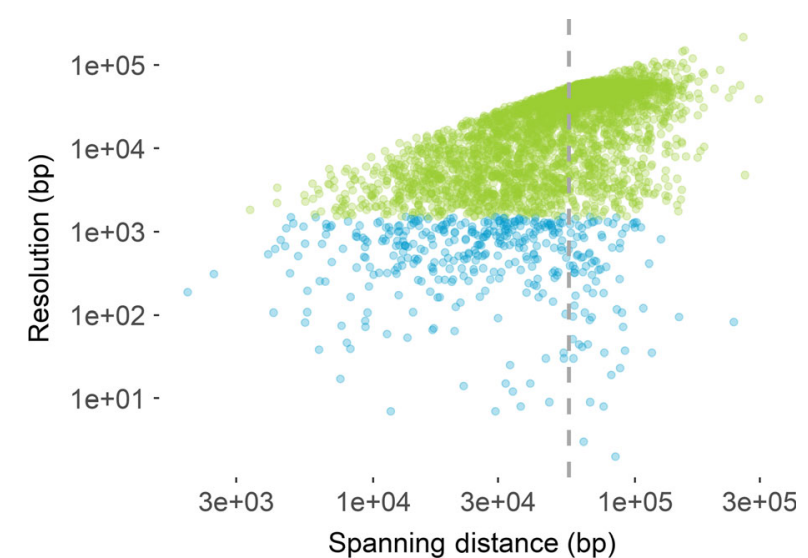

Figure 1. (a) Schematic representation of crossovers and gene conversions detected from haplotype shifts. Each molecule (m) consists of linked reads displayed as arrowed blocks connected by a horizontal line indicating their linkage. The red and green markers indicate the parental origin of the allele. Molecule $m_{1}$ represents a recombinant molecule showing phase shift marking a crossover. The resolution (res) of the crossover is defined as the length between two consecutive phase shifting markers, while spanning distance is the distance between the first and the last marker in the molecule. Molecule $m_{2}$ contains a gene conversion with two supporting markers with a minimal and maximal tract length denoted by $x$ and $y$, respectively. For GC detection the maximum track length ( $x$ ) was set to $400 \mathrm{bp}$. Molecule $m_{3}$ is non-recombinant, while in molecule $m_{4}$ the putative $\mathrm{CO}$ is discarded because its block length is shorter than 400 bp and displays only one phase shift. (b) Spanning distance versus crossover resolution with a CO represented either by a green or blue dot. Blue dots mark COs that were used to find correlations with sequence features. The dashed line represents the mean spanning distance of $55.9 \mathrm{~kb}$. 
(Figure 1a) from $1.9 \mathrm{~kb}$ to $297 \mathrm{~kb}$ (mean $55.9 \mathrm{~kb}$, Figures 1b and Figure S4a), respectively. The resolution of each molecule is negatively correlated with the coverage of reads overlapping a SNP marker (Pearson correlation coefficient $r=-0.59, P$-value $<2.2 \mathrm{e}-10$ ) (Figure $\mathrm{S} 4 \mathrm{~b}$ ), suggesting that increasing the sequence coverage would likely further improve the resolution.

A single homologous $\mathrm{CO}$ event results into two reciprocal recombinant molecules. When mapped against the reference genome, such a pair of overlapping recombinant molecules would display reciprocal haplotype profiles and point to the same phase shift position. However, currently we cannot precisely establish the actual total number of $\mathrm{CO}$ events from recombinant molecules. Sizes may differ between molecules because of random DNA breaks during library preparation and sequencing. Furthermore, molecules are randomly primed, and when sequenced, likely will differ in their read profiles. Also, recombinant molecules originating from multiple gametes and generated by multiple $\mathrm{CO}$ events, may still map to overlapping positions in the reference genome. In addition, we cannot identify the recombinant chromosome pairs that originate from a single gamete. These limitations prevented to link recombinant molecules with overlapping $\mathrm{CO}$ regions to a distinct number of $\mathrm{CO}$ events that generated them. Nonetheless, to determine the minimum number of $\mathrm{CO}$, we screened 3819 $\mathrm{CO}$ regions, and assigned sets of recombinant molecules with overlapping $\mathrm{CO}$ regions to individual recombination events. This left a minimum of 3169 putative $\mathrm{CO}$ recombination events. We then manually checked phase shift blocks for inconsistencies in 50 randomly selected candidate $\mathrm{CO}$ regions. Taking advantage of the Integrative Genome Viewer (IGV) displaying barcode (BX) and molecule identifier (MI) annotation tags called by LONG RANGER, we found a false positive rate of $2 \%$. Furthermore, the flanking sequences for all randomly selected $\mathrm{CO}$ regions showed an unambiguous BlastN hit to their corresponding position in the tomato reference genome. We therefore anticipated assigned $\mathrm{CO}$ s resulting from ambiguous read mappings less likely. We also found a significant overlap between COs in pollen and in RILs (Demirci et al., 2017) ( $P$-value $=8.44 \times 10^{-23}$, Fisher exact test $)$ using bedtools fisher. While our stringent filtering method greatly improved accurate detection, it likely decreased sensitivity though. Given the input gDNA, representing about 625 recombined F1 genome copies, and the previously reported average recombination rate in tomato of 1.8 per chromosome pair (Sherman and Stack, 1995), the theoretical number of COs in our pollen sample would be 13500 . The theoretical maximum number of $\mathrm{CO}$, when compared to 3169 detected COs in our pollen sample, suggests a considerable number of $\mathrm{CO}$ s were left undetected. However, considering that interspecies $\mathrm{CO}$ rates (i.e between the homeologous chromosomes of tomato and pimpinellifolium) can decrease to less than $20 \%$ compared to intraspecies recombination frequency (Canady et al., 2006; Demirci et al., 2017), the actual total number of COs in the F1 hybrid pollen presumably was lower. In the case of an interspecific hybrid, homoeologous chromosomes will to some extent be different in terms of genomic (repeat differences), and cytogenetic (heterozygosity for smaller structural chromosome variants) differences. Previously, chromosome structural differences have been revealed between $S$. lycopersicum and S. pimpinellifolium, including mismatched kinetochores foldbacks and other irregularities (Anderson et al., 2010a,b). Such differences between homeologous chromosomes lead to disturbances in the synapsis of the homoeologues, and hence may dramatically decrease formation of CO events. Depending on the species in the tomato clade used, genetic lengths of individual chromosomes have been found to vary substantially (Bernachii and Tanksley, 1997). In addition, strong variation in recombination frequency has been shown dependent on the sex identity of the gametes. In plants, male and female meiosis differ considerably. Less recombination for male gametes was found in an F1 plant from a backcross to both the $S$. lycopersicum and $S$. pennellii parents (De Vicente and Tanksley, 1991). In contrast, Drouaud et al. (2007) found an elevated recombination frequency in male gametes from Arabidopsis, whereas Philips et al. (2015) reported on a higher recombination frequency for female gametes of barley and showed recombination rates are temperature dependent. Thus, caution should be taken when comparing recombination frequencies and genetic map lengths such as for species in the tomato clade and for plants in general. Although the average frequency of $\sim 0.42 \mathrm{CO}$ s per recombined $\mathrm{F} 1$ pollen chromosome represents $23 \%$ of the interspecies CO frequency, it nevertheless falls within the range as previously determined for an F6 RIL population (Demirci et al., 2017).

\section{Variation in $\mathrm{CO}$ frequencies}

The frequency of COs between haploid nuclei can directly be compared since all genome copies originated from one F1 plant, eliminating a possible bias in recombination that would be due to differences in genetic background. To determine distributions, we assigned $\mathrm{COs}$ to $50 \mathrm{~kb}$ bins ordered along each of the 12 tomato chromosomes. We made a distinction between euchromatin and heterochromatin positions as previously established (Sherman and Stack, 1995; Demirci et al., 2017). As expected, COs were not uniformly distributed. In all chromosomes a higher frequency was clearly observed for euchromatin portions, while pericentromeric heterochromatin regions were mostly devoid of COs (Figure 2a,b). As previously suggested, the low number of $\mathrm{CO}$ s in pericentromere domains probably occurred within small euchromatin islands harbouring actively transcribed genes (Peters et al., 2009; The 
(a)

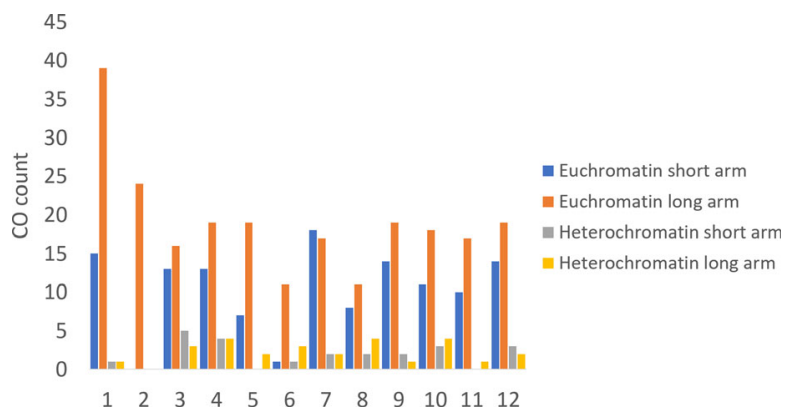

(b)

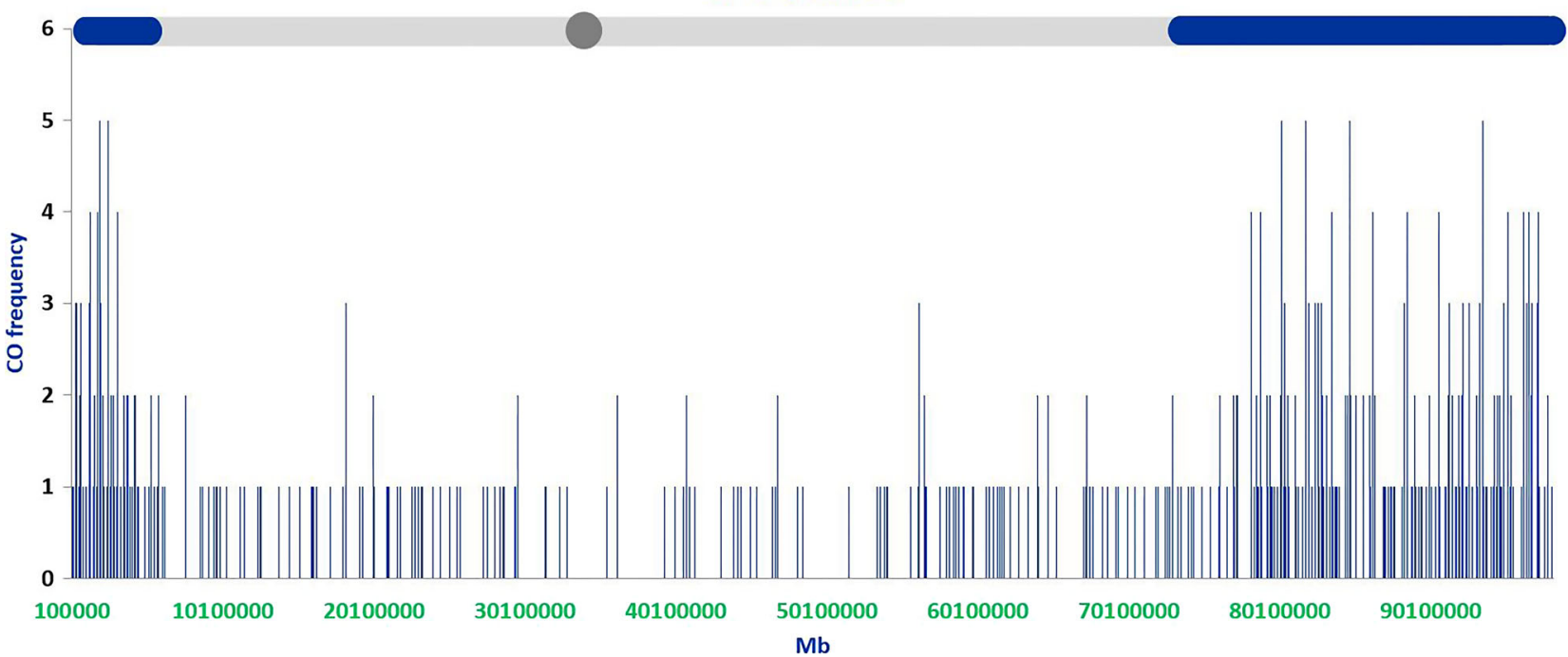

Figure 2. (a) Distributions of crossovers in the chromosome arms of pollen gametes. Positions of centromeres for division of chromosomes into long and short arms and euchromatin and heterochromatin sections were taken from Demirci et al. (2017). (b) Distribution of CO in pollen chromosome 1 in 100 kb bins. A schematic representation for tomato chromosome 1 is shown above the graph with euchromatin (dark blue) and heterochromatin (light grey), and centromere (dark grey).

Tomato Genome Consortium, 2012). To further measure the propensities of the F1 pollen chromosomes toward COs, we compared sequences of 283 high-resolution $\mathrm{CO}$ regions $(<1 \mathrm{~kb})$ against the tomato bacterial artificial chromosome (BAC) fingerprint map. These high resolution COs do not share overlapping flanking markers and thus were likely generated by independent recombination events. The sequence comparison yielded $167 \mathrm{CO}$ regions overlapping with tomato BACs anchored to the tomato EXPEN2000 genetic map. The order for 91 out 95 genetic markers associated with the anchored BACs coincided with the mapping order of all high-resolution $\mathrm{CO}$ s to the tomato reference genome, indicating it is unlikely that these COs were called from aberrantly mapped reads (Table S1). As expected, the frequency of $\mathrm{COs}$ in the heterochromatic regions is lower than in eurochromatic regions (Figure 2a,b). Furthermore, we observed a higher $\mathrm{CO}$ frequency for euchromatin regions toward the distal chromosome ends, except for chromosome 2 which showed a higher frequency towards the end of the long arm. These CO profiles in the F1 hybrid are in line with previously observed recombination profiles in tomato (Peters et al., 2009, 2012, Aflitos et al., 2014; Demirci et al., 2017, 2018).

\section{Correlation with primary sequence and gene features and hot and cold $\mathrm{CO}$ regions}

To obtain a detailed recombination profile, we assessed the sequence divergence and distribution of a subset of $400 \mathrm{CO}$ regions (Figure $1 \mathrm{~b}$ ). In order to retrieve a sufficient number of datapoints, we relaxed the resolution threshold to $1500 \mathrm{bp}$, which was $500 \mathrm{bp}$ lower compared to the threshold for genomic sequence feature enrichment detection. These COs regions are not overlapping and thus were also likely generated by independent recombination events. When comparing $\mathrm{CO}$ regions to the annotated reference genome, a permutation test showed a significantly higher rate of $\operatorname{COs}\left(P\right.$-value $\left.=1.0 \times 10^{-6}\right)$ in gene promoter regions (Figure $3 a$ ). This is in line with earlier results, reporting on $\mathrm{COs}$ biased toward regions upstream of transcription start sites (TSS) in tomato (Demirci et al., 2017), 
(a)

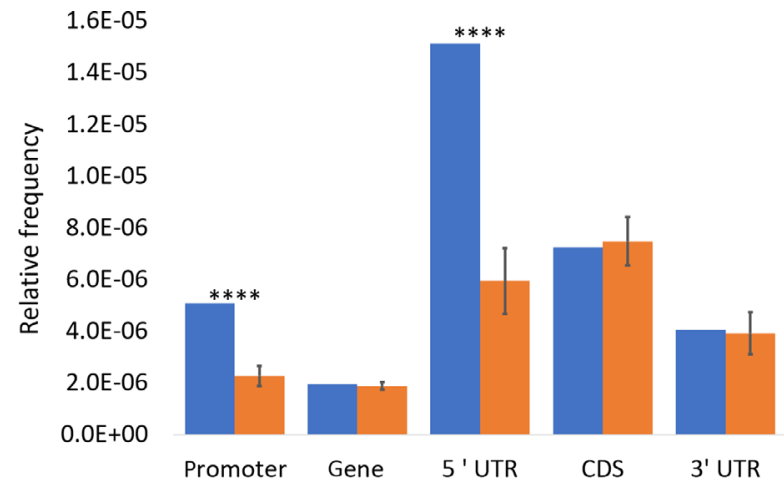

(b)
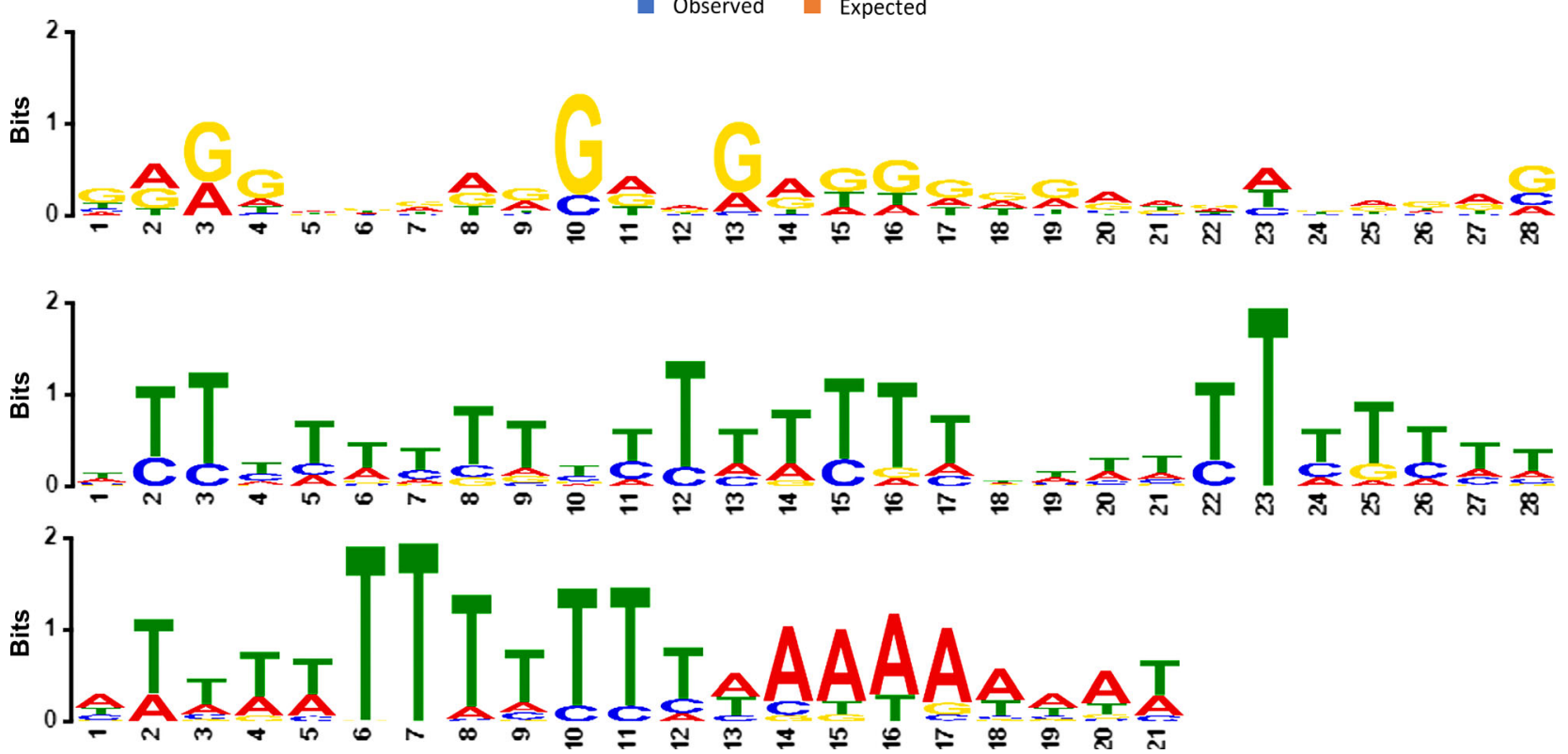

Figure 3. (a) Frequency distribution of COs observed in genic features. The observed and expected CO frequency per class is represented by blue and orange bars respectively. ${ }^{* * *} p<0.0001$. (b) Enriched sequence motifs in $\mathrm{CO}$ regions as discovered with MEME.

rice (Wu et al., 2003; Si et al., 2015; Demirci et al., 2018), maize (Rodgers-Melnick et al., 2015), and A. thaliana (Wijnker et al., 2013; Choi et al., 2018). To check whether the enrichment in the 5'-UTR is an artefact of skewed SNP density causing the $\mathrm{CO}$ regions to extend from the promoter to the 5'-UTR, we focused on a subset COs, overlapping either a promoter or $5^{\prime}$-UTR and excluded COs spanning both regions. The additional permutation test on this subset confirmed the enrichment of $\mathrm{COs}$ in the promoter (z-score $=2.29, P$-value $=0.0126)$ and $5^{\prime}$-UTR ( $z$-score $=2.04$, $P$-value $=0.0202$ ) (Figure S5). Moreover, using the MEME suite (Bailey et al., 2009), we identified significantly overrepresented sequence motifs in 283 high resolution (below $1 \mathrm{~kb}) \mathrm{CO}$ regions. The motifs were composed of poly-AG, poly- $T$ and poly(AT) sequences, occurring in 282 $\left(E=6.2 \times 10^{-24}\right), \quad 266 \quad\left(E=2.1 \times 10^{-20}\right) \quad$ and 280 $\left(E=9.0 \times 10^{-10}\right)$ CO regions, respectively (Figure $\left.3 \mathrm{~b}\right)$. Poly-T and poly(AT) signatures are typically found at noncoding and promoter regions, and often at transcription factor (TF) binding sites (O'Malley et al., 2016; Cherenkov et al., 2018) and have been found associated with crossovers for example in Arabidopsis (Choi et al., 2013; Wijnker et al., 2013; Shilo et al., 2015) and tomato (Demirci et al., 2017, 2018). Previously, we also found a weak correlation with AG dinucleotides (Demirci et al., 2017, 2018), further in line our observation of high $\mathrm{CO}$ incidence in gene promoter regions.

In addition, the observed distributions of recombinant sites along chromosomes suggested 'hot and cold regions'. Using $\mathrm{CO}$ density estimates, such regions with $\mathrm{CO}$ frequencies significantly deviating from the average were observed (Figure 4). We identified 23 hot regions in euchromatin portions except for chromosomes 3 and 6 . Furthermore, using a bedtools fisher test a significant number $\left(p\right.$-value $=4.77 \times 10^{-05}$, Fisher exact test $)$ of 15 out of 91 cold regions with a minimum length of $100 \mathrm{~kb}$ significantly overlapped with previously reported cold regions in F6 RILS (Demirci et al., 2017), further suggesting that 
Figure 4. Distribution of recombination sites in F1 tomato hybrid chromosomes. Heterochromatin, euchromatin, and centromeres are depicted as dark and light grey horizontal bars, and dark grey circles respectively. $\mathrm{CO}$ density graphs are superimposed on each chromosome. Crossover-dense (hot) and crossover-depleted (cold) regions in F1 hybrid pollen are shown as red and blue dots along each chromosome, respectively. Chromosome positions for NCO-GCs are indicated by pink triangles.

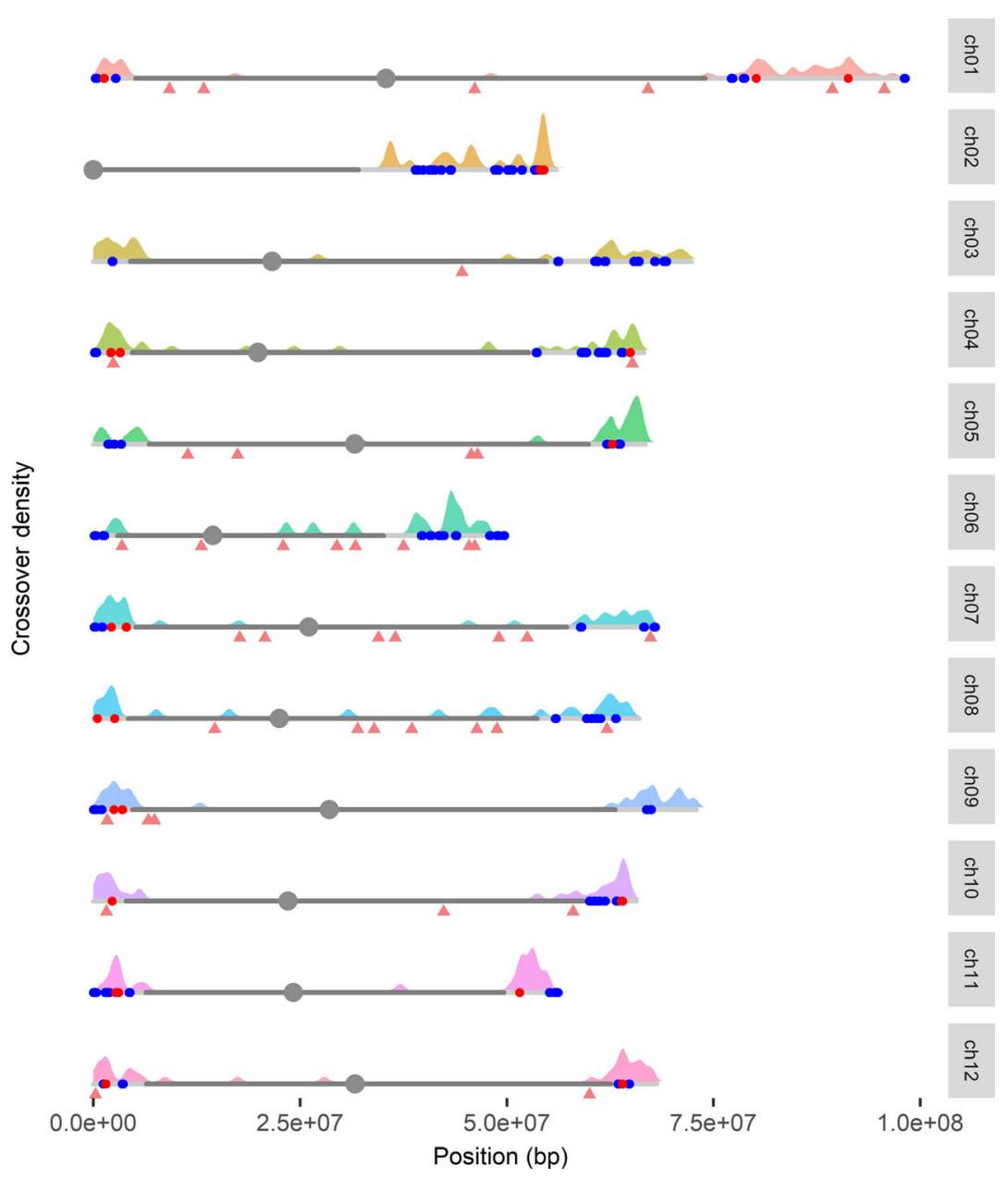

profiles in pollen gametes are representative for $\mathrm{CO}$ profiles observed in plants.

\section{Gene conversion designation}

Evidence has been presented for NCO-GC and CO-GCs in the animal and plant kingdom (Parniske et al., 1997; Xu et al., 2008; Duret and Galtier, 2009; Wijnker et al., 2013; Trombetta et al., 2016). When polymorphisms between alleles are involved, GCs in offspring alleles will be marked by SNPs, denoting the parental origin. We expected GCs also to occur during gametogenesis in our F1 tomato hybrid, though as far as we know a comprehensive genome wide analysis of GCs in interspecific tomato hybrids has not been presented until now. Both CO-GC and NCOGC would consist of a small segment, containing a few SNPs marking a phase shift. Indeed, GCs observed in other plants have been reported in the 25-400 bp range ( $\mathrm{Xu}$ et al., 2008; Wijnker et al., 2013). However, without knowing the recombinant pairs of chromosomes, our screening method currently can only detect NCO-GCs, occurring in small phase blocks less than $400 \mathrm{bp}$ (Figure S6). After selecting candidate GCs $<400 \mathrm{bp}$ and subsequent manual validation of supporting linked reads, we indeed detected 44 gene conversions within tracts between 35-369 bp. These 44 NCO-GCs do not share overlapping regions and thus were likely generated by independent recombination events. We prioritized on accuracy rather than sensitivity, by screening for small domains containing exactly one GC and at least 2 SNPs whose identity could be traced to the homologous donor allele. It is likely that the full chromosome complement is subject to GC and we indeed found footprints in all chromosomes. However, our stringent filtering did not leave GCs for chromosomes 2 and 11. We found read mappings at $3 \mathrm{GC}$ sites showing high quality SNPs (Phred-score $>30$ ) in addition to the expected polymorphisms that were detected between the parental homologous tomato and pimpinellifolium alleles. As these additional SNPs, occurring in a single read, could not be traced back to the parental alleles, we refer to them as 'non-parental' SNPs. The other linked reads generated from the same molecule flanking these non-parental SNPs mapped unambiguously to their homologous position. 
Using BLAST, we found for example a read with non-parental SNPs at a GC in chromosome 1, consisting of a segment specific for the target allele and another segment mapping to a nearby paralogous region in a reversed orientation approximately $250 \mathrm{bp}$ downstream of the conversion (Figures S7a and S7b). Considering that such clustered non-parental SNPs occurred in small segments identical to a parental paralogous allele, ectopic GC is the most parsimonious explanation. In addition, a separate set of 25 conversions that were reported as candidate GCs included insertions or deletions in a homopolymeric region. These indels, however, were not detected in the homologous or a paralogous allele. Currently, we cannot explain these indels by GC. We speculate they may have arisen as a result of illegitimate recombination such as non-homologous end-joining, or by polymerase slippage during replication, although sequencing errors of the homopolymeric region cannot be excluded.

\section{DISCUSSION}

Here we present an algorithm that faithfully detects phase shifts marking both meiotic crossovers and non-crossover associated allelic and ectopic gene conversions in haploid male gametes of a $S$. lycopersicum $\times S$. pimpinellifolium hybrid, based on $10 X$ Genomics linked read sequencing data. The algorithm identifies the parental origin of homozygous SNPs from linked reads of pollen gametes that have been mapped to the SL3.0 Heinz reference genome. The previously established high quality reference genomes of the S. lycopersicum cv. Heinz and S. pimpinellifolium parental lines (Aflitos et al., 2014) facilitated stringent filtering of false positives resulting from base calling or read mapping errors, and allowed for accurate $\mathrm{CO}$ and $\mathrm{GC}$ calls from phase shifts as presented here. Although, we couldn't establish the precise number of COs, because pairs of overlapping recombinant molecules do not have the same read profile and size, obscuring the identity of recombinant pairs in linked read data, we related non-overlapping recombinant molecules to a minimum number of $\mathrm{CO}$ events that generated them. Our analyses showed $\mathrm{CO}$ enrichment in promoter regions and the occurrence of specific sequence motifs consistent with previous results (Demirci et al., 2017, 2018). In addition, we now also show an elevated $\mathrm{CO}$ frequency in the $5^{\prime}$-UTR regions. Homologous meiotic recombination has been shown to occur around the transcription start sites and nucleosome-depleted regions, suggesting that open chromatin is accessible to diverse cellular machineries (Pan et al., 2011; Choi et al., 2013; Wijnker et al., 2013; Shilo et al., 2015; Demirci et al., 2017, 2018). A diversity analysis of 529 Arabidopsis TF-binding motifs showed that $25 \%$ have at least 4 consecutive $A / T$ stretches (O'Malley et al., 2016). These $A / T$ rich motifs have been found overrepresented for example in TF-binding sites related to auxin response in Arabidopsis
(Cherenkov et al., 2018). A relationship between transcribed genes and the proximity to recombination hotspots was reported for yeast (Mancera et al., 2008). Furthermore, AT-rich domains appear to disfavour nucleosome occupancy and recently have been shown to be involved in the formation of DSB and hotspots of recombination in Arabidopsis (Choi et al., 2018). The enhanced recombination in both promoter and $5^{\prime}$-UTR regions might thus be explained by the local structural conformation of the DNA that is accessible for replication, recombination, and DNA transcription complexes as previously suggested (Gottipati and Helleday, 2009; Demirci et al., 2017, 2018). The CO preference at promoter regions and $\mathrm{CO}$ hot and cold spots significantly overlapping with the regions of elevated and repressed $\mathrm{CO}$ in F6 RIL plants, further suggests that we faithfully detected COs and GCs in pollen gametes. It is possible that we have missed COs, especially in regions devoid of SNPs or with low coverage. However, since the observed recombination frequency in haploid spores was in the same range as previously observed for tomato hybrids (Demirci et al., 2017, 2018), we speculate that the number of missed COs is relatively low. Nevertheless, approximately $5 \times 10^{6}$ heterozygous SNPs and $4.7 \times 10^{5}$ indels have not been taken into consideration as potential $\mathrm{CO}$ markers yet, and may serve to further improve the resolution and sensitivity of CO and GC detection from linked read data. Additional adjustments, such as increased read coverage, and allowing for multiple phase shifts per molecule, also might improve the sensitivity. Although, we currently do not consider recombination events from multiple phase shifts, molecules with complex phase patterns in ranges between $30-100 \mathrm{~kb}$ were detected. We do not rule out complex GC in tomato, rather these observations hint at a tomato recombination machinery that is more complex than currently anticipated. Indeed, high-resolution mapping of meiotic crossovers and non-crossovers for example in yeast showed conversion tracts exhibiting complex patterns of genotype change, probably resulting from repair of multiple patches of heteroduplex, or alternatively, mismatch repair switching between conversion and restoration (Mancera et al., 2008; Leseque et al., 2013). Furthermore, the involvement of tomato transposable elements as drivers of meiotic recombination cannot be ruled out, since we observed putative GC in recombinant molecules overlapping with repeats and transposon copies. Previously, meiotic recombination and $\mathrm{GC}$ induced by the Mutator $(M u)$ transposon in maize, resulted in deletions affecting gene function of the knotted 1 gene (Mathern and Hake, 1997). A study on the trans-acting regulatory $M u D R$ transposon revealed increasing $\mathrm{GC}$ and intragenic meiotic recombination in the vicinity of a nonautonomous $\mathrm{Mu}$ transposon (Yandeau-Nelson et al., 2005). Furthermore, maize centromeres consisting of an abundant class Ty3/ Gypsy like transposable elements, known as Centomeric 
Retroelements, apparently exhibited widespread GC (Shi et al., 2010). We nevertheless currently prioritize on accuracy rather than sensitivity, excluding molecules overlapping with repeat and transposon copies to avoid aberrant recombination events called from misaligned recombinant molecules. This limitation could be overcome by lowering the molecule per GEM ratio and increasing the average length per molecule, sustaining faithful molecule mappings at a higher SNP levels, which then could shed more light on a possible role of transposons in meiotic $\mathrm{CO}$ recombination and $\mathrm{CO}$ associated and NCO associated GC in tomato.

Recombination profiling in plants has seen many techniques and approaches (Lambing and Heckmann, 2018). Among them are profiling strategies for male gametophytes which have relied on amplification of randomly amplified polymorphic DNA and single sequence repeat markers (Chen et al., 2008), tetrad analysis of Arabidopsis qrt mutants (Copenhaver et al., 2000; Wijnker et al., 2013), whole genome amplification (WGA) of DNA of male spores (Aziz and Sauve, 2008) in combination with FACS sorting and KASP genotyping (Dreissig et al., 2015), and recently also linked read sequencing of Arabidopsis $\mathrm{Col} \times$ Ler F1 gametes (Sun et al., 2019) and gametes from mouse and stickleback (Dreau et al., 2019). While linked-read sequencing of pollen gametes and sperm cells enabled highthroughput profiling of $\mathrm{COs}$, NCO related recombination events were not reported (Sun et al., 2019; Dreau et al., 2019). The genome coverages in these linked-read based profiling studies were considerably higher $(\sim 170 \times)$ compared to our genome coverage of approximately $100 \times$. We could not retrieve information on the map-based coverages in the aforementioned studies, which prevented further comparison to our map-based coverage of $68 x$.

The strategy described in this study has both fundamental and applied research implications. Its high throughput enables profiling of pooled recombination events, without the need to screen an offspring population. Our screening requires a pollen sample from a single F1 offspring plant, which makes the technology applicable for screening many parental breeding line combinations. Such screenings could for example provide guidance for selection of compatible donor and recipient genomes to overcome linkage drag problems. Since our method requires a single generation, it also becomes practical to profile recombination in crops with relatively long generation periods. Crossover recombination can now be assessed relatively fast and cheap with unsurpassed precision down to a resolution of $2 \mathrm{bp}$ as we have shown here. It thus becomes possible to assess the effects of genetic background, genome structure, and environmental conditions on recombination in greater detail and at a higher response level. This technology can thus boost breeding of advanced crops aimed at maintaining food security and safety, which is urgently needed in the face of the socio-economic and environmental challenges that traditional plant breeding currently faces (Nature Plants editorial, 2018; Nature editorial, 2018). Furthermore, recombination profiles from genetically diverse panels that are tested under variable environmental conditions can be used in a machine learning approach to improve our current CO prediction models (Demirci et al., 2018), providing further insight into gross similarities between species and species-specific aspects of recombination.

The current understanding of the recombination machinery in tomato is still incomplete as suggested by the complex patterns of exchanges that we detected. For example, besides 41 allelic GCs observed in both euchromatin and heterochromatin portions of the chromosomes, we detected 2 ectopic GCs in chromosome 1 and one in chromosome 3 in a reversed configuration relative to a nearby paralogous allele. We currently cannot explain the reversed configuration, but speculate that small inversions, as shown in mouse and Solanum hybrids, may cause local inversion loops in synapsed chromosomes (Borodin et al., 1991; Sherman and Stack, 1995; Anderson et al., 2010a,b). A DSB in such inversion loops may perhaps be resolved into a gene conversion with an inverted orientation relative to the donor allele. Interestingly, a number of studies report on intrachromosomal DSB repairs in plants which, depending on a direct-repeat or inverted-repeat configuration of the involved locus, were thought to have been processed via the single strand annealing (SSA) or synthesis-dependent strand-annealing (SDSA) pathway, resulting either in a deletion or a gene conversion respectively (reviewed in Puchta, 2005; Morrical, 2015). Furthermore, we observed 25 conversion tracts that cannot be explained by homologous CO or GC only. Careful breakpoint analysis showed that these recombinants contain indels that were not observed in the parental homologous or paralogous alleles. Our current analyses remain indecisive to the origin of these additional indels. These polymorphisms could have arisen for example by polymerase slippage during sequencing. Alternatively, these polymorphisms may have been introduced via alternative repair pathways such as non-homologous end joining (NHEJ) (Puchta, 2005). Indeed, micro-homology studies in human, have pointed at NHEJ and template-switching mechanisms generating small additional indels at repaired breaks (Kidd et al., 2010; Abyzov et al., 2015). Interestingly, interrupted GC was shown to generate truncated $M u$ elements in maize. The interruption was proposed to result from hybridizing direct repeats, upon which the gap resolution would lead to a deletion (Mathern and Hake, 1997). In our data we occasionally detected GCs associated with small deletions in recombinant molecules overlapping transposon related repeats. Whether transposon related repeats around these putative recombination events in tomato mediated 
deletions at repaired breaks, currently remains speculative though. Furthermore, NHEJ has been reported in yeast, mammals, and Drosophila to repair double stranded breaks by direct re-joining without extensive processing, frequently introducing unequal sequence exchanges such as indels (reviewed in Caridi et al., 2017). The recruitment of the HR or NHEJ pathway seems to be dependent on the chromatin compaction as has been observed in human where DSB in heterochromatin appears more prone to NHEJ than HR mediated repair (Caridi et al., 2017; Nisa et al., 2019). NHEJ might partly explain the conversions that we observed in the pericentromeric heterochromatin of F1 tomato hybrid chromosomes. Moreover, combinations of HR and NHEJ mediated repairs have been reported to contribute to a complex mosaic of exchanges at repaired breaks (Puchta, 2005). Recently, it was demonstrated that DSB in repetitive 45S rDNA loci from Arabidopsis, during meiosis are shielded from $\mathrm{HR}$ and preferentially repaired via NHEJ (Sims et al., 2019). Whether the occurrence of such additional indel markers can be attributed to the aforementioned NHEJ mediated repair mechanism during meiotic recombination in tomato, currently remains speculative and will be subject to further study.

\section{EXPERIMENTAL PROCEDURES}

\section{Selection of tomato accessions}

An F1 plant was produced from an interspecific cross between S. Iycopersicum cv. Heinz 1706 and S. pimpinellifolium CGN14498, which was used as the male and female parent respectively as described in Aflitos et al. 2014. The F1 plant was grown to flowering in a greenhouse under normal day night light ( $16 \mathrm{~h} / 8 \mathrm{~h})$ conditions at $24^{\circ} \mathrm{C}$.

\section{High molecular weight DNA isolation from pollen}

Mature pollen was released from flowers using a handheld milk frother device and collected in a $1.5 \mathrm{ml}$ tube. Pollen were then stored at $4^{\circ} \mathrm{C}$ at a relative humidity of $32 \%$ or immediately germinated after harvesting according to the protocol described by $\mathrm{Lu}$ et al. 2015 with modifications. Approximately $2-10 \mathrm{mg}$ pollen were covered with a small gauze and positioned in a small petri dish containing saturated $\mathrm{NA}_{2} \mathrm{HPO}_{4}$ for $4-8 \mathrm{~h}$ at $25^{\circ} \mathrm{C}$ without bringing the pollen into direct contact with the solution. The hydrated pollen were then incubated in $2.5 \mathrm{ml}$ germination medium $\left(20 \mathrm{~mm}\right.$ MES, $3 \mathrm{~mm} \mathrm{Ca}\left(\mathrm{NO}_{3}\right)_{2} .4 \mathrm{H}_{2} \mathrm{O}, 1 \mathrm{~mm} \mathrm{KCl}, 0.8 \mathrm{~mm}$ $\left.\mathrm{MgSO}_{4} .7 \mathrm{H}_{2} \mathrm{O}\right), 1.6 \mathrm{~mm}$ Boric acid $\left(\mathrm{H}_{3} \mathrm{BO}_{3}\right), 24 \%(\mathrm{w} / \mathrm{v})$ PEG $4000,2.5 \%$ $(\mathrm{w} / \mathrm{v})$ sucrose at $\mathrm{pH} 6.0$ for $1.5 \mathrm{~h}$ at $25^{\circ} \mathrm{C}$, shaking at $90 \mathrm{rpm}$ in the dark. Germination was monitored by assessing small samples with a light microscope at $20 \times$ magnification and was terminated when pollen tubes reached approximately $2 \times$ the diameter of the pollen grain. Germinated pollen were isolated from the medium with a $20 \mu \mathrm{m}$ cell strainer (pluriStrainer, pluriSelect Life Sciences, Leipzig, Germany), collecting the flow through in a $50 \mathrm{ml}$ tube by washing with $1 \mathrm{ml}$ nuclei isolation buffer $(0.3 \mathrm{~m}$ sucrose, $10 \mathrm{~mm}$ $\mathrm{NaCl}, \quad 10 \mathrm{~mm}$ 2-(N-morpholine)-ethanesulphonic acid, $5 \mathrm{~mm}$ ethylenediamine-tetraacetic acid, $0.2 \mathrm{~mm}$ spermine, $0.5 \mathrm{~mm}$ spermidine, $0.2 \mathrm{~mm}$ phenylmethylsulphonyl fluoride, $5 \mathrm{~mm}$ dithiothreitol, and $0.2 \%(\mathrm{v} / \mathrm{v})$ Triton-X-100, adjusted to $\mathrm{pH} 7.4$ with $2 \mathrm{~m}$
$\mathrm{NaOH})$. Nuclei were subsequently isolated from pollen according to Gao et al. (2013) with modifications. Subsequently, nuclei were collected by placing the $20 \mu \mathrm{m}$ pluriStrainer with a connector ring on top of a $10 \mu \mathrm{m}$ pluriStrainer and then put on top of a clean $50 \mathrm{ml}$ tube. The $20 \mu \mathrm{m}$ cell strainer was used to isolate the released nuclei and the non-germinated pollen from the germinated pollen, while the $10 \mu \mathrm{m}$ cell strainer was used to separate the released nuclei from the non-germinated pollen. Next $1 \mathrm{ml}$ nuclei isolation buffer was added to the pollen sample and nuclei were subsequently released by gentle homogenisation of the pollen tubes using a pestle for cell strainer (Sigma-Aldrich, Zwijndrecht, The Netherlands) at $60-100 \mathrm{rpm}$ for $1 \mathrm{~min}$. Nuclei were rinsed from the $10 \mu \mathrm{m}$ pluriStrainer and collected in a $50 \mathrm{ml}$ tube and centrifuged three times at $500 \mathrm{~g}$ for $5 \mathrm{~min}$ to pellet the nuclei. The nuclei were then embedded in $2 \%$ liquid low melting point agar kept at $45^{\circ} \mathrm{C}$. The pellet was suspended in $10 \mu \mathrm{l}$ nuclei isolation buffer, raising the temperature gently to $45^{\circ} \mathrm{C}$ before adding $20 \mu \mathrm{l}$ of $2 \%$ Low Melting Point (LMP) agarose at $45^{\circ} \mathrm{C}$ and casting the solution into a mould. Plugs containing the nuclei can either be stored in TE buffer at $4^{\circ} \mathrm{C}$, or directly be used for high molecular weight DNA isolation from the plugs according to the BioNano Genomics extraction protocol 'IrysPrep Plug Lysis Long DNA isolation' (30026D) as described by the manufacturer. The DNA concentration and molecule length was determined with a Qubit (Thermo Fisher Scientific, Waltham, MA, USA) and a DNA Fragment Analyzer (Agilent, Santa Clara, CA, USA).

\section{X Genomics library preparation and sequencing}

The 10X Genomics libraries were constructed with the Chromium $^{\mathrm{TM}}$ Genome Reagent Kits v2 (10X Genomics ${ }^{\circledR}$ ) according to the Chromium ${ }^{\mathrm{TM}}$ Genome v2 Protocol (CG00043) as described by the manufacturer. Sequencing was carried out using Illumina HiSeq 2500 with $2 \times 150$ bp paired-end reads to a genome coverage of approximately 100X. The LONG RANGeR pipeline from 10X Genomics was used to process the sequencing output and align the reads to the tomato reference genome (The Tomato Genome Consortium, 2012). The LouPE genome browser summary view from 10X Genomics was used to retrieve the metrics on input DNA, sequencing, molecule length, coverage and gel beads in emulsion (GEM) performance. The number of F1 hybrid genome copies that were assessed was calculated using Lander-Waterman statistics (Lander and Waterman, 1988).

\section{Sequence alignment and data processing}

Illumina PE sequences from RIL parent S. pimpinellifolium CGN 14498 and from $S$. Iycopersicum cv. Heinz from the 150 Tomato Genome Project (Aflitos et al., 2014; Demirci et al., 2017) were aligned to the $S$. lycopersicum cv. Heinz reference genome v3.0 using the read mapper tool from CLC Genomics workbench v11.0.1 (https://www.qiagenbioinformatics.com/). Reads were subsequently realigned to adjust for local mapping inconsistencies near insertions and deletions using the local read mapping alignment tool from CLC and the indel realignment GATK toolkit v.4. Homozygous and heterozygous single nucleotide variants (SNPs and indels) where then called using GATK UnIFIEDGENOTYPER and the fixed ploidy level variant detection algorithm from CLC separately. For GATK we used recommended parameter settings as described (https://software.broadinstitute.org/gatk/documentation/ article.php?xml:id=3225), whereas for the CLC fixed ploidy detection algorithm we used the following settings: Ploidy $=2$; Required variant probability $(\%)=80.0$; Ignore positions with coverage above $=100$ 000; Ignore broken pairs $=$ Yes; Minimum coverage $=8$; Minimum count $=2$; Minimum frequency $(\%)=20.0$; 
Neighborhood radius $=5$; Minimum central quality $=20$; Minimum neighborhood quality $=15$; Relative read direction filter $=$ Yes; Significance $(\%)=1.0$. Homozygous S. pimpinellifolium SNPs predicted by both GATK and CLC were then intersected and selected as segregating markers, excluding all positions found to be polymorphic between the tomato parent and the reference genome. Chromium barcoded sequences from the pollen sample were processed and aligned to the $S$. lycopersicum cv. Heinz v3.0 reference genome (https://solgenomics.net/organism/Solanum_lyc opersicum/genome) using the LONG RANGER pipeline with default parameter settings. The output was processed by SAMTOOLS v1.3 calmd to generate the MD tags and then filtered per chromosome. Paired end (PE) reads without barcodes and molecule IDs, having a mapping quality below 60 , showing an abnormal template length larger than $2 \mathrm{~kb}$, or discordantly aligned to multiple chromosomes, were removed. The resulting alignment file was intersected with the list of $S$. pimpinellifolium homozygous SNPs to retrieve reads relevant to the phase shift detection. Reads having identical barcode (BX), molecule identifier (MI) and alignment start position were marked as clonal reads and discarded. Both CIGAR and MD tags of non-clonal reads were then parsed to assign the parental origin of the polymorphism that matched a segregating marker. The haplotype for each linked read molecule was determined using the pipeline illustrated in Figure S1. Briefly, putative haploblock shifts were assigned to a linked read molecule if two adjacent SNP blocks, consisting of at least three uninterrupted and contiguous SNP markers, were assigned to different parents, i.e. 'aaabbb' or 'bbbaaa'. CO recombination sites were then called taking the midpoint position between the two SNP markers flanking the phase shift. Molecules with candidate COs were subsequently filtered against tomato repeats (ITAG3.2_Repeat Modeler_repeats), chloroplast insertions in the tomato nuclear genome, and tomato retrotransposons (tomato_retrotransposons_PRI v2) retrieved from ftp://ftp.solgenomics.net/genomes/Solanum_lyc opersicum/repeats. To avoid aberrant haplotype shifts due to misalignment of tandem repeats aligned molecules overlapping at least $10 \%$ in length with repeats or candidate CO regions having a read coverage of flanking markers higher than $95 \%$ of all marker coverages across the genome were discarded (Figure S2). To focus on the unambiguous occurrences of GC, molecules with multiple haplotype shifts were excluded. A block of at least two SNPs with the same parental origin flanked by SNPs from the other parent were identified as gene conversion, i.e. 'aaabbaaa' or 'bbbaabbb'. Molecules with phased regions flanked by two phase shifts were selected as candidate gene conversions (GCs). Candidate GCs were retained if (i) they were at most $400 \mathrm{bp}$ long, supported by SNPs having a high base quality score (Phred-score $>30$ ) matching with either parental allele, (ii) the supporting reads were not soft/hard-clipped, (iii) and did not overlap parental indel markers (to avoid false positives from aberrant indel marker calls). Remaining GCs containing indels that were not present in either homologous parental allele, but with supporting SNPs located within 3 bp distance of the indel, or GCs containing indels within homopolymeric regions of at least $4 \mathrm{bp}$ were reported separately as they may have arisen from non-homologous directed repair mechanisms. Read alignments were visualized in the Intergrative Genome Browser (IGV) (Robinson et al., 2011) and used to manually examine the linked read support for randomly selected $\mathrm{CO}$ s and GCs, including a check on consistent $\mathrm{BX}$ and $\mathrm{MI}$ identifiers, and a verification of the SNP identity with a Phred score 30 or higher matching to either parental allele.

\section{Sequence motif discovery and gene feature distribution}

Sequences of $1 \mathrm{~kb}$, flanking both sides of the midpoint of high resolution $(<1 \mathrm{~kb})$ CO regions $(n=283)$ were selected for motif discovery with the MEME suite using default settings (Bailey et al., 2009). Only overrepresented motifs at least 6 bp long, occurring in at least $50 \mathrm{CO}$ regions, were reported. To find possibly enriched gene ontology terms, motifs with significant $E$-value $<0.05$ were submitted to GOMo (Buske et al., 2010).

\section{Identification of hot and cold regions (CO-dense and CO-poor regions)}

Using the R-package function 'density', we estimated the CO density using a Gaussian kernel (bandwidth $=20000 \mathrm{nt}$ ) for every 1000 nt. Euchromatic and heterochromatic regions were delimited as previously described (Demirci et al., 2017). We then computed the $p$-value for local maxima and minima detected by the $\mathrm{R}$ function 'turnpoints' based on density estimates in random recombination region sets generated by shuffling the observed euchromatic crossovers in each chromosome 10000 times. Significant $p$-values of density peaks or hot regions were adjusted for multiple testing using a Bonferroni correction with a 0.05 cut-off threshold. For density pits or cold regions, we apply the $P$-value cut-off of 0.05 without Bonferroni correction and merged consecutive pits that are at most $10 \mathrm{~kb}$ apart. Only cold regions covering at least $100 \mathrm{~kb}$ were reported.

\section{Genetic marker order}

Sequences from linked reads containing haplotype shifts, marking a potential crossover recombination, were compared against marker sequences from the Tomato-EXPEN-2000.v1 genetic map (https://solgenomics.net) using BLASTN and then filtered using an identity threshold of $98 \%$. Positional marker orders between the linked read genetic map and the tomato genetic maps were compared to assess marker collinearity.

\section{ACKNOWLEDGEMENTS}

The work presented here is supported by the MEICOM Marie Sklodowska-Curie Innovative training Network (ITN), H2020-MSCAITN-2017 Horizon 2020 Grant agreement number 765212.

\section{AUTHOR CONTRIBUTIONS}

Roven Rommel Fuentes and Sander A. Peters conceived and designed the analysis. Thamara Hesselink; Elio Schijlen, Ronald Nieuwenhuis, and Willem van Dooijeweert collected the data. Roven Rommel Fuentes, Aalt D.J. van Dijk, Dick de Ridder, and Sander A. Peters contributed data or analysis tools. Roven Rommel Fuentes, Linda Bakker, Ronald Nieuwenhuis, Sander A. Peters performed the analysis. Roven Rommel Fuentes, Sara Diaz Trivino, Jorn R. de Haan, Gabino Sanchez Perez, Xinyue Zhang, Paul Fransz, Hans de Jong, Aalt-Jan van Dijk, Dick de Ridder, and San$\operatorname{der} A$. Peters wrote the paper.

\section{CONFLICT OF INTEREST}

The authors declare not to have conflicts of interests concerning this publication. 


\section{DATA AVAILABILITY STATEMENT}

All the data used in this study are available from the corresponding author upon request.

\section{SUPPORTING INFORMATION}

Additional Supporting Information may be found in the online version of this article.

Figure S1. Schematic representation of the crossover and gene conversion discovery pipeline.

Figure S2. Distribution of marker coverage and counts.

Figure S3. Variant classes of $S$. pimpinellifolium compared to $S$. lycopersicum parental genomes.

Figure S4a. Spanning distance versus crossover resolution with CO represented by a green dot.

Figure S4b. Correlation of molecule coverage and resolution.

Figure S5. Enrichment of COs in promoter and 5'-UTR regions.

Figure S6. Schematic representation of recombinant chromosomes with $\mathrm{CO}$ s and GCs.

Figure S7a. Possible configurations involved in an ectopic gene conversion in chromosome 1.

Figure S7b. Sequence alignment of a linked read to repetitive alleles marking an ectopic GC in chromosome 1.

Table S1. Comparison of positional orders between crossovers and TOMATO-EXPEN 2000 genetic map markers.

\section{REFERENCES}

Abyzov, A., Li, S., Kim, D.R. et al. (2015) Analysis of breakpoints from 1092 humans reveals details of mutation mechanisms. Nat. Comm. 6, 7256.

Aflitos, S., Schijlen, E., de Jong, H. et al. (2014) Exploring genetic variation in the tomato (Solanum section Lycopersicon) clade by whole-genome sequencing. Plant J. 80, 136-148.

Aflitos, S.A., Sanchez-Perez, G., De Ridder, D., Fransz, P., Schranz, M.E., De Jong, H. and Peters, S.A. (2015) Introgression browser: high-throughput whole-genome SNP visualization. Plant J. 82, 174-182.

Anderson, L.K., Covey, P.A., Larsen, L.R., Bedinger, P. and Stack, S.M. (2010a) Structural differences in chromosomes distinguish species in the tomato clade. Cytogenet. Genome Res. 129, 24-34.

Anderson, L.K., Covey, P.A., Larsen, L.R., Bedinger, P. and Stack, S.M. (2010b) Structural differences in chromosomes distinguish species in the tomato clade. Cytogenet Genome Res. 129, 24-34.

Aziz, A.N. and Sauve, R.J. (2008) Genetic mapping of Echinacea purpurea via individual pollen DNA fingerprinting. Mol. Breeding. 21, 227-232.

Bailey, T.L., Boden, M., Buske, F.A., Frith, M., Grant, C.E., Clementi, L., Ren, J., Li, W.W. and Noble, W.S. (2009) Meme suite: tools for motif discovery and searching. Nucleic Acids Res. 37, W202.

Baurens, F.-C., Martin, G., Hervouet, C. et al. (2019) Recombination and large structural variations shape interspecific edible bananas genome. Mol. Biol. Evol. 36, 97-111.

Bernacchi, D. and Tanksley, S.D. (1997) An interspecific backcross of Lycopersicon esculentum $\times$ L. hirsutum : Linkage analysis and a QTL study of sexual compatibility factors and floral traits. Genetics, 147, 861-877.

Borodin, P.M., Gorlov, I.P., Agulnik, A.I., Agulnik, S.I. and Ruvinsky, A.O. (1991) Chromosome pairing and recombination in mice heterozygous for different translocations in chromosomes 16 and 17. Chromosoma, 101, 252-258.

Borts, R.H., Chambers, S.R. and Abdullah, M.F.F. (2000) The many faces of mismatch repair in meiosis. Mutation Res. 451, 129-150.

Buske, F.A., Bodén, M., Bauer, D.C. and Bailey, T.L. (2010) Assigning roles to DNA regulatory motifs using comparative genomics. Bioinformatics, 26, 860-866.

Canady, M.A., Ji, Y. and Chetelat, R.T. (2006) Homeologous rrecombination in Solanum lycopersicoides introgressions lines of cultivated tomato. Genetics, 174, 1775-1788.
Caridi, P.C., Delabaere, L., Zapatoczny, G. and Chiolo, I. (2017) And yet, it moves: nuclear and chromatin dynamics of a heterochromatic doublestrand break. Philos. Trans. R. Soc. B, 372, 20160291.

Chen, P.-H., Pan, Y.-B. and Chen, R.-K. (2008) High-throughput procedure for single pollen grain collection and polymerase chain reaction in plants. J. Int. Plant Biol. 50, 375-383.

Cherenkov, P., Novikova, D., Omelyanchuk, N., Levitsky, V., Grosse, I., Weijers, D. and Mironova, V. (2018) Diversity of cis-regulatory elemnts associated with auxin response in Arabidopsis thaliana. J. Exp. Bot. 69, 329-339.

Choi, K., Zhao, X., Kelly, K.A. et al. (2013) Arabidopsis meiotic crossover ho spots overlap with H2A.Z nucleosomes at gene promoters. Nat. Genet. 45, 1327-1338

Choi, K., Zhao, X., Tock, A.J. et al. (2018) Nucleosomes and DNA methylation shape meiotic DSB frequency in Arabidopsis thaliana transposons and gene regulatory regions. Genome Res. 28, 532-546.

Copenhaver, G.P., Keith, K.C. and Preuss, D. (2000) Tetrad analysis in higher plants. A budding technology. Plant Physiol. 124, 7-16.

De Vicente, M.C. and Tanksley, S.D. (1991) Genome wide reduction of backcross progeny derived from male versus female gametes in an interspecific cross of tomato. Theor. Appl. Genet. 83, 173-178.

Demirci, S., Van Dijk, A.D.J., Sanchez Perez, G., Aflitos, S.A., De Ridder, D. and Peters, S.A. (2017) Distribution, position and genomic characteristics of crossovers in tomato recombinant inbred lines derived from an interspecific cross between Solanum lycopersicum and Solanum pimpinellifolium. Plant J. 89, 554-564.

Demirci, S., Peters, S.A., De Ridder, D. and Van Dijk, A.D.J. (2018) DNA sequence and shape are predictive for meiotic crossovers throughout the plant kingdom. Plant J. 95, 686-699.

Dreau, A., Venu, V., Avdievich, E., Gaspar, L. and Jones, F.C. (2019) Genome-wide recombination map construction from single individuals using linked read sequencing. Nat. Commun. 10, 4309. https://doi.org/10.1038/ s41467-019-12210-9.

Dreissig, S., Fuchs, J., Cápal, P., Kettles, N., Byrne, E. and Houben, A. (2015) Measuring meiotic crossovers via multi-locus genotyping of single pollen grains in barley. PLoS ONE, 10, e0137677.

Drouaud, J., Mercier, R., Chelysheva, L., Bérard, A., Falque, M., Martin, 0. Zanni, V., Brunel, D. and Mézard, C. (2007) Sex-specific crossover distributions and variations in interference level along Arabidopsis thaliana chromosome 4. PLoS Genet. 3, e106.

Duret, L. and Galtier, N. (2009) Biased gene conversion and the evolution of mammalian genomic landscapes. Annu. Rev. Genomics Hum. Genet. 10, 285-311.

Filler Hayut, S., Melamed Bessudo, C. and Levy, A.A. (2017) Targeted recombination between homologous chromosomes for precise breeding in tomato. Nat. Commun. 8, 15605.

Ganal, M.W., Durstewitz, G., Polley, A. et al. (2011) A Large maize (Zea mays L.) SNP genotyping array: development and germplasm genotyping, and genetic mapping to compare with the B73 reference genome. PLOS ONE, 6, e28334.

Gao, Y.B., Wang, C.L., Wu, Y., Wu, J., Zhou, H.S. and Zhang, S.L. (2013) A method to isolate male gametic nuclei from pear pollen tubes. J. Hortic. Sci. Biotechnol. 88, 313-319.

Gottipati, P. and Helleday, T. (2009) Transcription-associated recombination in eukaryotes: link between transcription, replication and recombination. Mutagenesis, 24, 203-2010.

Havekes, F.W.J., De Jong, J.H., Heyting, C. and Ramana, M.S. (1994) Synapsis and chiasma formation in four meiotic mutants of tomato (Lycopersicum esculentum). Chromosome Res. 2, 315-325.

Kidd, J.M., Graves, T., Newman, T.L. et al. (2010) A human genome structural variation sequencing resource reveals insights into mutational mechanisms. Cell, 143, 837-847.

Lambing, C. and Heckmann, S. (2018) Tackling plant meiosis: from model research to crop improvement. Front. Plant Sci. 9, 829.

Lander, E. and Waterman, M. (1988) Genomic mapping by fingerprinting random clones: a mathematical analysis. Genomics, 3, 231-239.

Leseque, Y., Mouchiroud, D. and Duret, L. (2013) GC-biased gene conversion in yeast is specifically associated with crossovers: molecular mechanisms and evolutionary significance. Mol. Biol. Evol. 30, 1409-1419.

Lu, Y., Wei, L. and Wang, T. (2015) Method to isolate a large amount of generative cells, sperm cells and vegetative nuclei from tomato pollen for "omics" analysis. Front. Plant Sci. 6, 391. 
Mancera, E., Bourgon, R., Brozzi, A., Huber, W. and Steinmetz, L.M. (2008) High-resolution mapping of meiotic crossovers and non-crossovers in yeast. Nature, 454, 479-486.

Mathern, J. and Hake, S. (1997) Mu element-generated gene conversions in maize attenuate the dominant knotted phenotype. Genetics, 147, 305314.

McKenna, A., Hanna, M., Banks, E., et al. (2010) The genome analysis toolkit: a MapReduce framework for analyzing next-generation DNA sequencing data. Genome Res. 20, 1297-1303.

Mercier, R., Mézard, C., Jenczewski, E., Macaisne, N. and Grelon, M. (2015) The molecular biology of meiosis in plants. Annu. Rev. Plant Biol. 66, 297-317.

Moens, P.B. (1964) A New interpretation of meiotic prophase in Lycopersicon Esculentum (Tomato). Chromosoma, 15, 231-42.

Morrical, S.W. (2015) DNA-pairing and annealing processes in homologous recombination and homology-directed repair. Cold Spring Harb. Perspect. Biol. 7, a016444.

Nature Editorial. (2018) The cost of climate inaction. Nature, 561, 433

Nature Plants Editorial. (2018) Technologies to boost breeding. Nat. Plants, $4,1$.

Nisa, M.-U., Huang, Y., Benhamed, M. and Raynaud, C. (2019) The plan DNA damage response: signaling pathways leading to growth inhibition and putative role in response to stress conditions. Front. Plant Sci. 10 653.

O'Malley, R.C., Huang, S.-S.C., Song, L. et al. (2016) Cistrome and epicistrome features shape the regulatory DNA landscape. Cell, 165, 1280 1292.

Pan, J., Sasaki, M., Kniewel, R. et al. (2011) A hierarchical combination of factors shapes the genome-wide topography of yeast meiotic recombination initiation. Cell, 144, 719-733.

Paran, I., Rouppe van der Voort, J., Lefebvre, V. et al. (2004) An integrated genetic linkage map of pepper (Capsicum spp.). Mol. Breed. 13, 251-261.

Parniske, M., Hammond-Kosack, K.E., Golstein, C., Thomas, C.M., Jones, D.A., Harrison, K., Wulff, B.B. and Jones, J.D. (1997) Novel disease resistance specificities result from sequence exchange between tandemly repeated genes at the Cf-4/9 locus of tomato. Cell, 91, 821-832.

Peters, S.A., Bargsten, J.W., Szinay, D., Van de Belt, J., Visser, R.G.F., Bai Y. and De Jong, H. (2012) Structural homology in the Solanaceae: analysis of genomic regions in support of synteny studies in tomato, potato and pepper. Plant J. 71, 602-614.

Peters, S.A., Datema, E., Szinay, D. et al. (2009) Solanum lycopersicum cv. Heinz 1706 chromosome 6: distribution and abundance of genes and retrotransposable elements. Plant J. 58, 857-869.

Phillips, D., Jenkins, G., Macaulay, M., Nibau, C., Wnetrzak, J., Fallding, D., Colas, I., Oakey, H., Waugh, R. and Ramsay, L. (2015) The effect of temperature on the male and female recombination landscape of barley. New Phytol. 208, 421-429.
Puchta, H. (2005) The repair of double-strand breaks in plants: mechanisms and consequences for genome evolution. J. Exp. Bot. 56, 1-14.

Robinson, J.T., Thorvaldsdóttir, H., Winckler, W., Guttman, M., Lander, E.S., Getz, G. and Mesirov, J.P. (2011) Integrative genome viewer. Nat. Biotech. 29, 24-26.

Rodgers-Melnick, E., Bradbury, P.J., Elshire, R.J., Glaubitz, J.C., Acharya C.B., Mitchell, S.E., Li, C., Li, Y. and Buckler, E.S. (2015) Recombination in diverse maize is stable, predictable, and associated with genetic load. Proc. Natl Acad. Sci. USA, 112, 3823-3828.

Sherman, J.D. and Stack, S.M. (1995) Two-dimensional spreads of synaptonemal complexes from Solanaceous Plants. Genetics, 141, 683-708.

Shi, J., Wolf, S.E., Burke, J.M., Presting, G.G., Ross-lbarra, J. and Kelly, R.D. (2010) Widespread gene conversion in centromere cores. PLoS Biol. 8 , e1000327.

Shilo, S., Melamed-Bessudo, C., Dorone, Y., Barkai, N. and Levy, A.A. (2015) DNA crossover motifs delineate open chromatin regions in Arabidopsis. Plant Cell, 27, 2427-2436.

Si, W., Yuan, Y., Huang, J. et al. (2015) Widely distributed hot and cold spots in meiotic recombination as shown by the sequencing of rice F2 plants. New Phytol. 206, 1491-1502.

Sims, J., Copenhaver, G.P. and Schlögelhofer, P. (2019) Meiotic DNA repair in the nucleolus employs a nonhomologous end-joining mechanism. Plant Cell, 31, 2259-2275.

Sun, H., Rowan, B.A., Flood, P.J. et al. (2019) Linked-read sequencing of gametes allows efficient genome-wide analysis of meiotic recombination. Nat. Commun. 10, 4310. https://doi.org/10.1038/s41467-019-12209-2

The Tomato Genome Consortium. (2012) The tomato genome sequence provides insights into fleshy fruit evolution. Nature, 485, 635-641.

Trombetta, B., Fantini, G., D'Atanasio, E., Silletto, D. and Crucianin, F. (2016) Evidence of extensive non-allelic gene conversion among LTR elements in the human genome. Nat. Sci. Rep. 6, 28710.

Van Os, H., Andrzejewski, S., Bakker, E. et al. (2006) Construction of a 10 000-marker ultradense genetic recombination map of potato: providing a framework for accelerated gene isolation and a genomewide physical map. Genetics, 173, 1075-1087.

Wijnker, E., Velikkakam James, G., Ding, J. et al. (2013) The genomic landscape of meiotic crossovers and gene conversions in Arabidopsis thaliana. Elife, 2, e01426.

Wu, J., Mizuno, H., Hayashi-Tsugane, M. et al. (2003) Physical maps and recombination frequency of six rice chromosomes. Plant J. 36, 720-730.

Xu, S., Clark, T., Zheng, H., Vang, S., Li, R., Wong, G.K.-S., Wang, J. and Zheng, X. (2008) Gene conversion in the rice genome. BMC Genom. 9, 93.

Yandeau-Nelson, M.D., Zhou, Q., Yao, H., Xu, X., Nikolau, B.J. and Schnable, P.S. (2005) MuDR transposase increases the frequency of meiotic crossovers in the vicinity of a $M u$ Insertion in the maize a1 gene. Genetics, 169, 917-929. 